\title{
Caracterización psicológica de la pasión romántica
}

\section{Psychological characterization of romantic passion}

\author{
Rozzana Sánchez Aragón ${ }^{1} \bowtie$ \\ ${ }^{1}$ Universidad Nacional Autónoma de México
}

$\triangle$ Av. Universidad 3000, Col. Copilco-Universidad, Del. Coyoacán, C.P. 04510, Edificio C Cubículo 23 PB. Ciudad de México, México. Correo electrónico: rozzara@unam.mx

Recibido: 2 de marzo de $2017 \quad$ Aprobado: 11 de julio de $2018 \quad$ Disponible en línea: 1 de octubre del 2018
Cómo citar este artículo: Sánchez Aragón R. (2018). Caracterización psicológica de la pasión romántica. Pensando Psicología,
14(24). doi: https://doi.org/10.16925/pe.v14i24.XXXX

\section{Resumen}

Introducción: la pasión romántica como universal cultural posee matices particulares que definen sus diferentes fases: atracción, enamoramiento, amor obsesivo y desesperado Aunado a su conceptualización, existen variables que se relacionan a su experiencia, como las premisas histórico-socioculturales que crean expectativas sobre ésta, los atributos de personalidad sexual percibida en el objeto de pasión, las conductas y estrategias de seducción utilizadas durante el evento pasional, así como la satisfacción sexual resultante de su consumación.

Objetivo: la presente investigación tuvo como propósito caracterizar a cada una de las fases de la pasión con base en las variables relacionadas a su experiencia.

Método: se trabajó con una muestra no probabilística de 495 participantes residentes de la Ciudad de México entre 15 y 58 años que reconocieron haber vivido pasión romántica.

Resultados: luego de unos análisis de correlación, producto momento de Pearson, se lograron identificar los efectos comunes a los participantes en la descripción de su experiencia para las fases, así como la forma diferencial de sentir pasión romántica por hombres y mujeres.

Conclusión: la presente investigación logró ampliar la concepción de las fases del amor pasional con base en los datos relativos a las Phsc's, personalidad sexual, estrategias de seducción y la satisfacción sexual en una muestra mexicana. Asimismo, se identificó lo que distingue a hombres y mujeres respecto a la experiencia de dichas fases y se discute conforme a las normas y creencias de la cultura mexicana.

Palabras claves: cultura, obsesión, pasión romántica, personalidad sexual, seducción. 


\title{
Psychological characterization of romantic passion
}

\begin{abstract}
Introduction: the romantic passion as a cultural universal has particular nuances that define its different phases: attraction, falling in love, obsessive and desperate love. Along with its conceptualization, there are variables that relate to its experience, such as the historic-socio-cultural premises that create expectations about it, the attributes of sexual personality perceived in the passion object, the behaviors and strategies of seduction used during the passionate event, as well as the sexual satisfaction resulting from its consummation.

Objective: the purpose of this research was to characterize each of the passion phases based on the variables related to its experience.

Method: we worked with a non-probabilistic sample of 495 residents of Mexico City between 15 and 58 years old who recognized having lived a romantic passion.

Results: after some Pearson's correlation analysis, we identify the effects common to the participants in the description of their experience for the phases, as well as the differential way of feeling romantic passion for men and women.

Conclusion: the present investigation was able to broad the conception of the phases of passionate love based on the data related to the PHSC'S, sexual personality, seduction strategies and sexual satisfaction in a Mexican sample. Likewise, what distinguished men and women regarding the experience of these phases was identified and discussed according to the norms and beliefs of the Mexican culture.
\end{abstract}

Keywords: culture, obsession, romantic passion, sexual personality, seduction.

\section{Resumo}

Introdução: romântico como paixão cultural universal tem nuances específicas que definem suas diferentes fases: atração, paixão, amor obsessivo e desespero. Além de sua conceituação, existem variáveis que se relacionam com a sua experiência, como SCHPs que criam expectativas sobre este a personalidade sexual percebida atribui 0 objeto da paixão, comportamentos e estratégias de sedução utilizados durante 0 evento apaixonado e a satisfação sexual resultante de sua consumação.

Objetivo: esta pesquisa foi caracterizar cada uma das fases da paixão com base em variáveis relacionadas com a sua finalidade experiência.

Método: trabalhou com uma amostra não-probabilística de 495 participantes residentes da Cidade do México entre 15 e 58 anos admitiu ter vivido paixão romântica.

Resultados: após uma análise de correlação do momento do produto Pearson, ser capaz de identificar os participantes efeitos comuns em descrever sua experiência para as fases e a forma como diferencial a sentir paixão romântica para homens e mulheres.

Conclusão: Esta pesquisa foi capaz de expandir a fase de concepção de amor apaixonado com base em dados relativos aos PHSC'S, personalidade sexual, estratégias de sedução e satisfação sexual em uma amostra mexicana. Também identificou o que distingue homens e mulheres quanto à experiência dessas fases é discutida de acordo com as normas e crenças da cultura mexicana.

Palavras-chave: cultura, obsessão, paixão romântica, personalidade sexual, sedução. 


\section{Introducción}

La pasión romántica (PR) de acuerdo con Jankowiak (1995) es cualquier atracción intensa que involucra la idealización del otro en un contexto erótico. La idealización conlleva el deseo por la intimidad y la expectativa placentera de que la experiencia se mantenga por cierto tiempo - desconocido- en el futuro. Así, la PR está en agudo contraste con la fase del amor de compañía - a veces conocido como apego-, que se caracteriza por el cambio gradual $-\mathrm{y}$ muchas veces desapercibido inicialmente - en una relación más pacífica $-\mathrm{o}$ menos urgente-, más cómoda -0 menos intensa-, y más satisfactoria - o menos estática-. El amor en esta etapa se basa en una reciprocidad fuerte y permanente de afecto, atención y respeto que deriva de una asociación a largo plazo (Hatfield, 1988). En palabras de Collins y Gregor (1995) el amor de compañía es más un asunto de placer sencillo que de éxtasis.

Así, la PR ha sido concebida durante décadas en formas aparentemente distintas, pero claramente traslapadas que evidencian el acuerdo entre autores. En los años setenta, Lee (1976) la define como una atracción inmediata y poderosa hacia el otro, seguida de un periodo de miedo y tensión; luego de conocimiento, disfrute y éxtasis para declinar hacia el desencantamiento. Durante esta experiencia se tienen síntomas fisiológicos tales como: excitación, shock, reacciones químicas, transpiración, presión estomacal, incremento de la respiración y del pulso cardiaco. Walster y Walster (1978) lo ven como un estado salvajemente emocional, una confusión de sentimientos, ternura y sexualidad, alegría y pena, ansiedad y alivio, altruismo y celos. Durante los años ochenta, Brehm (1985) indica que la pasión es una intensa experiencia sexual que representa la combinación de la imaginación y emoción, y sirve para motivar al ser humano a construir una mejor visión del mundo. Por su parte, Sternberg (1986) dice que es toda emoción que guía a la atracción y consumación sexual en una relación romántica. En los años noventa, Fletcher y Kininmonth (1991) la señalan como la excitación y todo lo involucrado en las relaciones sexuales, mientras que Fehr (1993) la interpreta más ampliamente como un elixir mágico que hace que el ser humano se sienta realizado en la vida, implica prisa y precipitación por el placer en su versión más perfecta y un anhelo por la pasión erótica, creando con ello un éxtasis vertiginoso que brinda momentos de felicidad apasionada, euforia, excitación, risa y satisfacción. Para
Hatfield y Rapson (1996) es una emoción caliente, frecuentemente considerada aplastante, amor obsesivo, enfermedad del amor, estar locamente enamorado, encaprichamiento o estar enamorado. Un estado de intenso anhelo y deseo de unión con el otro.

Para la primera década del siglo xxi Sánchez Aragón (2000) la definió como un desbordamiento de emociones basadas en el deseo sexual intenso por consumarlo en la entrega sin medida e incluye: enamoramiento, la necesidad de estar cerca del otro constantemente, irracionalidad, amor, ímpetu, la intrusión obsesiva, la búsqueda desesperada por tener cercanía física continua, la alegría, el erotismo y la sensualidad y reacciones como sentir "mariposas en el estómago". Más tarde, Troy (2005) concluye que, para experimentar PR y su inherente excitación, la persona debe exceder su experiencia pasada en cierto modo para que el efecto fisiológico y subjetivo sea más intenso.

Aunque en general se identifica el lado positivo de la PR, el hecho de que esté basada en la excitación y en una intensa emoción que comprende altas y bajas emocionales, cuyo desencadenamiento se da rápidamente, pero tiende a disminuir con el tiempo; facilita que su experiencia se torne obscura. Al respecto, Villanueva Orozco (2004) identificó a los celos, posesión, dependiencia, miedo de perder a la persona, ansiedad, desesperación, incertidumbre, tristeza, dolor, nostalgia, desilusión, frustración, enojo, arrebatos, pleitos, falta de control y concentración, descuido de actividades y extrañamiento como aspectos negativos de la pasión.

Por su parte, Hatfield (1988) reconoce ambos lados de la PR y establece que cuando el amor es recíproco y se siente unión con el otro, éste se asocia con realización y éxtasis; mientras que cuando el amor no es correspondido y se siente alejamiento, se vincula con vacío, ansiedad o desesperanza.

Con base en lo anterior, Sánchez Aragón (2007a) planteó y posteriormente comprobó la existencia de un continuo de la PR que incluye cuatro fases que se definen de la siguiente manera:

1. Atracción: es una etapa de valencia positiva que se caracteriza por la felicidad, goce, emoción, ilusión, plenitud sentida ante el pensamiento o presencia de la persona por la cual se empieza a sentir enamoramiento. Asimismo, refleja el gusto y gran placer por procurar cuidar y hacer planes con la otra persona, pues ésta satisface sus necesidades emocionales, de autoestima, cumple expectativas $\mathrm{y}$ brinda romance. 
2. Enamoramiento: es conceptualizada como el deseo intenso de explorar, tocar, relacionarse sexualmente, la seducción y el estremecimiento surgen ante la idea o presencia del objeto de pasión (OP), en donde el fantasear e inquietarse por la posibilidad de ver a la persona estimulan la sensualidad y el antojo.

3. Amor obsesivo: versa sobre: a) la autopercepción de angustia, tristeza y desesperación ante el alejamiento o separación del ser amado, la persona siembra toda expectativa de felicidad, tranquilidad y valía en la atención, contacto o correspondencia del otro; y b) la invalidez experimentada ante la ausencia del objeto de amor, misma que se manifiesta en la ansiedad, urgencia de ver a la persona, agonía, inseguridad, miedo y deseos de no vivir.

4. Amor desesperado: aborda la búsqueda persistente de interacción con el OP, incluyendo estrategias de persecución, obligarle a hacerle compañía, acorralarle, imponiéndole su presencia, comprometiéndole a compartir espacios, presionándole, bromeándole sobre temas sexuales con el fin de impedir que algo se interponga entre ellos y así lograr la atención o correspondencia.

No obstante los avances en torno al entendimiento de lo que es la PR y sus fases - que Sánchez Aragón (2007b) reconoció- los esfuerzos continúan para lograr una caracterización más clara de este constructo. Para ello hay que reconocer - de entrada- que la PR ha sido catalogada como un universal cultural (Jankowiak y Fischer, 1992), un fenómeno complejo y emocionalmente multifacético cuya experiencia se ve definida por aspectos de orden: 1) cultural (Hinton, 1993; Jankowiak, 1995; Person, 2007) que especifican el valor de la PR para ese grupo; 2) individual (Hatfield y Rapson, 1996; Person, 2007) pues hay formas diferenciales de interesarse en ella dependiendo del sexo, el momento del ciclo de vida en el cual se experimente y la particular forma de vivir las emociones, cogniciones y generar conductas inherentes a ésta; y 3) diádico (Sánchez Aragón, 2007b) ya que es un constructo que involucra a dos personas que interactúan y en quienes la $\mathrm{PR}$ puede surgir. Con este contexto claro, es necesario ahondar en estos aspectos para luego examinar empíricamente su carácter multifacético.

\section{La cultura: normas y valores de la pasión}

Jankowiak (1995) mencionó que la pasión romántica es considerada un universal cultural y esto lo confirma cuando al reanalizar sus datos encuentra que las historias de amor encontradas en 166 grupos culturales distintos, tenían una falta de especificidad cultural. Es decir, todas sonaban igual sin importar si provenían de americanos o europeos, de un polígamo mormón o de un inuit o taita o mangaian. Así que cuando se reconoce que la cultura es la encargada de regular los sentimientos y las ideas, jerarquizar las relaciones interpersonales, estipular los tipos de papeles sociales que se deben seguir y las reglas de interacción entre los individuos (Díaz Guerrero, 1994; Lucker, 2002), la experiencia de la PR está incluida.

Desde la perspectiva de Díaz Guerrero (1994) la forma en la se operacionaliza a la cultura es a través de las premisas histórico-socioculturales (Phsc) y es por medio de ellas que se pueden reconocer aquellas normas y creencias que rigen el comportamiento de los miembros de un grupo. Así, cuando se trata de identificar las Phsc de la PR, Sánchez Aragón, Jiménez Rodríguez y Martínez Cruz (2013) encontraron que - para los mexicanos - cuando alguien siente PR debe: vivirse en forma intensa, las personas deben estar dispuestos a entregarse en todos los sentidos, realizar su deseo, dejando fluir su emoción y hacer uso de la imaginación para satisfacer su pasión plena y recíprocamente. Asimismo, se debe fantasear, soñar, imaginar y pensar nuevas formas de realizar sus deseos sexuales con la persona amada y así crear momentos especiales, llenos de seducción y placer. De acuerdo a estas autoras, la PR exige que las personas se besen, abracen y acaricien; pero se debe meditar, reflexionar, identificar y pensar respecto a la importancia, naturalidad y bondad de experimentar dicha emoción. Aunque se le considera una emoción digna de disfrute sin restricciones, se debe ejercer cierto control a través de pensamientos respecto a sus consecuencias.

Un correlato de la PR que también se ve mediatizado por la cultura (Hatfield, Rapson y Martel, 2010) es el deseo sexual y en conjunto son consideradas las emociones más poderosas que, además de organizarse alrededor de ciertos criterios culturales y psicológicos, compiten directamente en la vida del individuo. Al respecto Jankowiak (1995) indica que cada cultura prioriza o la sexualidad o el amor, pero mezclarlas resulta complicado, no obstante, Hatfield y Rapson (1996) reconocen el uso intercambiable de los términos de PR y deseo sexual. Ante esto, si la PR es vista como "un intenso anhelo de unión con el otro" el deseo sexual es "un intenso anhelo de unión sexual con el otro" (p. 3); lo cual hace que la pasión 
y el deseo se encuentren fusionados de tal forma que es muy dificil separarlos. En concordancia, al evaluar la experiencia subjetiva de la PR, Sánchez Aragón (2006) encontró que ésta se componía de tres factores: uno positivo que implica ternura, confianza e ilusión, un negativo en donde hay incertidumbre, angustia y ahogo; y uno sexual que abarca a la excitación, el deseo y el erotismo. Estos hallazgos muestran la inevitable alianza entre la PR y su correlato sexual, pues impacta a la satisfacción sexual referida posteriormente como un elemento diádico. Asimismo, hay que señalar que la PR y su vivencia subjetiva también se verá influida por los atributos de personalidad de sus interlocutores.

\section{El individuo: personalidad sexual}

Esta amalgama entre la pasión romántica y la sexualidad es mencionada por Shopenhauer (citado en Jankowiak, 1995), quien decía que el amor romántico es "un truco" que la naturaleza le juega al ser humano para asegurar la reproducción” (p. 7). Justamente aquí es pertinente abordar a la personalidad sexual, la cual - desde el punto de vista evolutivo- se refiere a los atributos del individuo involucrados en el apareamiento humano ya que afectan el ejercicio de la sexualidad y la selección de la pareja (Schmitt y Buss, 2000). Estos autores crearon una medida denominada los Sexy Seven que reúne las características de la propia personalidad sexual, como: atractivo sexual, exclusividad en la relación, orientación de género, restricción sexual, disposición erotofílica, involucramiento emocional y orientación sexual. Más tarde en México, Sánchez Aragón, Cruz Martínez y Retana Franco (2006) modificaron y validaron dicha medida con el fin de evaluar no solo la personalidad sexual propia sino también la percibida en su objeto de pasión (OP), además de examinar las diferencias sexuales en torno a sus factores. Entre las diferencias entre sexos se obtuvo que los hombres se evaluaron como más caballerosos, conquistadores, promiscuos y perversos; y percibían a las mujeres más puras e inmaculadas. Por otra parte, ellas se ven más involucradas emocionalmente, lo cual se ve apoyado por las Phsc en torno a la experiencia de la atracción y la pasión en la cultura mexicana. Más recientemente, Cruz Vargas (2018) validó nuevamente la versión de la personalidad sexual percibida en el OP encontrando mayor claridad en sus factores. Como se entrevé, la personalidad sexual tiene un papel importante no solo en la experiencia romántico-pasional de los miembros de la pareja, sino en la forma en que estos se eligen. A continuación se revisarán aspectos individuales que entran en juego en la dinámica diádica y que involucran el intercambio de emociones, conductas de seducción; y a partir de ello, la evaluación satisfactoria de la vida sexual.

\section{La diada: comunicación emocional, estrategias de seducción y satisfacción sexual}

Durante la interacción, la pareja romántica o pasional hace uso de la comunicación para expresar tranquilidad, deseo, y necesidad. Las relaciones son inherentemente un fenómeno comunicativo y dependiendo de su eficacia, el vínculo se puede ver enriquecido o empobrecido teniendo consecuencias en su calidad y repercución en su satisfacción (Guerrero, Andersen y Afifi, 2017).

Específicamente, la comunicación emocional se enfoca en la expresión e interpretación de mensajes desde los más insignificantes hasta aquellos vitales para la relación (Guerrero et al., 2017) que se interpretan a partir del tipo de vínculo existente y que generan placer o displacer. Para evaluar este tipo de comunicación, Sánchez Aragón (2012) desarrolló una medida en la que se estima qué tanto se experimentan una serie de emociones -incluyendo la pasión- a partir de los comportamientos comunicativos verbales y no verbales de la pareja. Entre las conductas que realiza la persona A que favorecen la experiencia de la pasión en la persona $B$, se encuentran: sugerencias sexuales, la creación de un ambiente sensual, poner una película erótica, contar sus fantasías, mostrar su cuerpo desnudo, hablarle al oído, besarla intensamente, abrazarla y acariciarla, todo con el fin de intensificar el momento.

Como se puede ver, la comunicación emocional de la pasión empieza a utilizarse como una herramienta para atraer a la otra persona, y esto es parte de lo que se realiza cuando se le desea seducir (Caldíz y Resnicoff, 1997). Así, la seducción es una forma de atracción, erotismo y placer que favorece la comunicación, la novedad y el sexo, así como el acercamiento y conocimiento de la pareja. Se percibe como una fuente de poder y una oportunidad de expresarse libremente y tanto a hombres como a mujeres el uso de su cuerpo les permite expresar su deseo, convirtiéndose a sí mismos en una práctica cotidiana de manifestación emocional (Sanz, 1995). En cuanto a la identificación de las estrategias de seducción en 
México, Carrasco Chávez (2010) reconoce cuatro: 1) expresión sexual, 2) búsqueda de cercanía y permanencia con la pareja, 3) desenvolvimiento y expresión libre, y 4) enamoramiento.

Como parte inseparable de la ejecución de las tácticas de seducción se encuentra la satisfacción sexual, misma que ha sido definida como la evaluación subjetiva o actitud sobre las conductas sexuales propias de la pareja (Álvarez Gayou, 1987), el cumplimiento de un "deseo erótico", "la manifestación final de la pasión", "aplacar un sentimiento", "sentirse complacido y amado" (Paredes y Pinto, 2009); o bien, como una respuesta afectiva que surge a partir de la propia evaluación subjetiva de la relación sexual (Byers 2005). De acuerdo a Young, Denny, Young y Luquis, (2000), Ziherl y Masten (2010) la satisfacción sexual comprende: la actividad sexual, el rendimiento - que incluye la frecuencia de actividad-, variedad de conductas sexuales, las actitudes positivas hacia la sexualidad, y la calidad de la relación.

Por su parte, García Rodríguez (2007) menciona que las dimensiones de la satisfacción sexual tienen que ver con los aspectos: físicos - el conocimiento del propio cuerpo y del compañero sexual, sensaciones experimentadas en las zonas erógenas, frecuencia, variedad, ejecución sexual, obtención del orgasmo-, psicológicos - aceptación de la propia sexualidad, sensación de plenitud, intimidad emocional, pensamientos y fantasías eróticas, expectativas sexuales, PR, comunicación emocional-y socioculturales - estresores sociales-situacionales, normas, mitos, valores, actitudes y creencias respecto al papel que los sexos deben jugar y lo que deben experimentar durante el encuentro sexual-. A diferencia de esta autora, Hudson (1997) había señalado que la satisfacción sexual era unidimensional a pesar de haber operacionalizado aspectos como: gozo, presión, evasión y aburrimiento. No obstante, al aplicar su escala en México (Cruz Vargas, 2018) identificó dos factores: satisfacción e insatisfacción.

En lo tocante a las diferencias entre hombres y mujeres en satisfacción sexual, Yela (2000) encontró que en los hombres la satisfacción sexual se relaciona con el tipo y duración de la relación de pareja, la satisfacción amorosa, permisividad sexual, celos sexuales y con el balance percibido entre compromiso e independencia. Mientras que en las mujeres se asocia con la PR y erótica, a la comunicación abierta, la satisfacción en general con la relación, los celos emocionales y las actitudes religiosas. En contraste, un estudio realizado por Metts y Cupach (1991), y años más tarde por Valdés, Sapién, y Córdoba (2004) reportan que la gratificación sexual en los hombres es una cuestión física relacionada con la frecuencia, el deseo, la excitación, el vigor, la ejecución o técnica así como alcanzar siempre el orgasmo. Mientras que para las mujeres, aunque se presentaba el orgasmo como señal de satisfacción sexual, los indicadores principales fueron las caricias, la intimidad, el amor y la estabilidad de la relación, primando la conexión emocional al placer sexual.

Se toma en consideración la propuesta de Sánchez Aragón (2007b) respecto a las fases de la pasión romántica y las contribuciones que la cultura, el individuo y la diada pueden hacer hacia una mejor definición de éstas. La presente investigación se encaminó a examinar las relaciones entre las fases de la PR — atracción, enamoramiento, amor obsesivo y amor desesperado - con las Phsc de la PR, la personalidad sexual del OP, la comunicación emocional, las estrategias de seducción y la satisfacción sexual en hombres y mujeres de la Ciudad de México para así caracterizar cada fase.

\section{Método}

\section{Participantes}

Se trabajó con una muestra no probabilística de 416 participantes (203 mujeres y 213 hombres) residentes de la Ciudad de México entre 19 y 40 años (X=22.63), cuyos niveles de escolaridad fueron: 75 de preparatoria, 333 de licenciatura y 4 de posgrado, 4 no respondieron. El 100 \% reconoció haber sentido pasión por alguien que era su pareja romántica estable, amigo o novio principalmente.

\section{Instrumentos}

\section{Escala multifásica del amor pasional en su versión}

corta (Sánchez Aragón, 2007b): dicha prueba es un autoreporte conformado por treinta reactivos en formato de respuesta tipo Likert de cinco puntos indicando grados de acuerdo a través de los cuales se evalúa en qué fase del continuo del amor pasional se sitúa cada individuo. La escala se compone de cuatro factores, a saber:

- Atracción que incluye reactivos como: y yo nos complementamos.

Me causa felicidad hacer planes con 
- Enamoramiento contiene ítems como:

Explorar el cuerpo de me excita. Fantaseo con

- Amor obsesivo se operacionaliza con oraciones como:

No estoy tranquilo si no sé dónde está todo el tiempo.

Tengo una necesidad fuera de lo normal de estar con

- Amor desesperado se compone de:

Siento que no me corresponde porque no se ha dado cuenta de que me ama.

Perseguir a es la única forma de obtener contacto con él/ella.

Sus respectivos coeficientes de confiabilidad Alpha de Cronbach son: .64, .79, .85 y .74. y su validez fue obtenida por medio de un análisis factorial en el que los factores explicaron el 54-50\% de la varianza.

Inventario de premisas histórico-socio-culturales de expresión emocional de la pasión romántica en su versión corta (Sánchez Aragón et al., 2013): escala conformada por treinta reactivos en formato tipo Likert que indican cinco grados de acuerdo organizados en seis factores que explicaron el $52.74 \%$ de la varianza. Se da una instrucción general ante la cual los participantes responden: "cuando las personas experimentan pasión romántica deben [...]” y a continuación se presentan los ítems. Los factores son: expresión abierta y plena $(\partial=.93)$ con ítems como abrazar y besar a la persona, estar dispuesto a entregarse y realizar su deseo. El segundo factor titulado deseo e imaginación $(\partial=.89)$ tiene reactivos como fantasear con la persona amada, soñar con tener contacto íntimo con la persona, e imaginarse haciendo de todo con la persona. Por su parte el factor contacto físico intenso $(\partial=.85)$ tiene oraciones como "estar junto a la persona amada lo más posible", expresar su emoción con abrazos y expresar su emoción con besos. El cuarto factor reflexión emocional $(\partial=.82)$ contiene reactivos como meditar en lo importante que es lo que sienten, reflexionar sobre la felicidad que experimentan y pensar que su emoción es natural. El quinto factor llamado disfrute $(\partial=.82)$ es evaluado por: disfrutar la emoción sin restricciones, sólo disfrutarla y dejar fluir la emoción con libertad. Finalmente, el factor control $(\partial=.73)$ se mide a través de: pensar en las consecuencias, ser responsable y cuidarse, y controlarse.
Inventario de personalidad sexual (Cruz Vargas, 2018). Esta versión se aplicó para estimar desde el punto de vista del participante- los rasgos que caracterizan a su OP. Consta de 51 reactivos que explicaron el $52.43 \%$ de la varianza y en donde quien responde indica qué tanto le caracteriza el rasgo en un escalamiento tipo Likert de cinco puntos. Los factores con sus coeficientes Alpha de Cronbach y ejemplos de sus reactivos se dan a continuación: promiscuo $(\alpha=.91)$-infiel, mujeriego-, involucrado emocionalmente $(\alpha=.88)$ —adorable, amoroso-, atractivo sexual ( $\alpha=.89)$ - apasionado, atrayente-, dispuesto eróticamente $(\alpha=.83)$ - conquistador, lujurioso-, orientación de género $(\alpha=.80)$ - masculino, hembra—, restricción sexual ( $\alpha=.73)$ — casto, religoso-.

Escala de comunicación emocional de las relaciones románticas (Sánchez Aragón, 2012): dicha escala consta de 97 ítems y su formato de respuesta es tipo Likert pictórico de cuatro caras que reflejan cuatro niveles de expresión emocional del amor-felicidad, pasión, celos, miedo, tristeza, enojo, sorpresa positiva, sorpresa negativa, sin embargo, para los fines de este estudio únicamente se utilizó el factor de pasión (ver figura 1) que se compone de 17 reactivos y cuyo coeficiente alpha de Cronbach es de .93, la media es de 2.80 y su DE de .75. Algunos ejemplos son: Si mi pareja me acaricia el cuerpo y Cuando mi pareja me abraza sugiriéndome que quiere algo más.

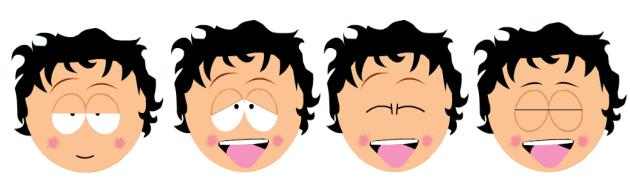

Figura 1. Formas de respuesta del factor de pasión de la escala de comunicación emocional de las relaciones románticas.

Fuente: elaboración propia.

Escala de estrategias de seducción femenina en su versión corta (Carrasco Chávez 2010): es un autoreporte dirigido a evaluar las formas de seducir de mujeres casadas y solteras, conformado por veinte reactivos en un formato tipo Likert con cinco opciones de respuesta ( $1=$ nunca a $5=$ siempre), cuyo estímulo para los reactivos fue: "Cuando deseo atraer a mi pareja para tener un momento íntimo con ella, yo [...]". Enseguida aprecen los reactivos que se encuentran distribuidos en cuatro factores que explican el $58.97 \%$ de la varianza expresión sexual $(\alpha=.97)$ que incluye reactivos como: acaricio sus genitales, le provoco sensaciones placenteras y lo excito. Búsqueda de 
cercanía y permanencia con la pareja $(\alpha=.92)$ comprende: llamo su atención, mantengo su interés y le coqueteo. El factor desenvolvimiento y expresión libre $(\alpha=.88)$ tiene ítems como: soy yo mismo, me demuestro seguro y me muestro libre de prejuicios. Por último el factor de enamoramiento $(\alpha=87)$ con oraciones como: soy amable, soy cariñoso, y hago una cena romántica.

Î́ndice de satisfacción sexual (Hudson, 1997; Cruz Vargas, 2018). Cuenta con 24 reactivos en formato de respuesta tipo Likert de cinco puntos que indican grados de acuerdo. Están encargados de evaluar la calidad del intercambio sexual mediante dos factores que al examinar su validez explicaron el $55.47 \%$ de la varianza satisfacción $(\alpha=.78)$ con 10 reactivos como "nuestra vida sexual es muy excitante" y "disfruto de las técnicas sexuales que usa o le gustan"; e insatisfacción $(\alpha=.93)$ con 14 reactivos como "cuando tenemos sexo es demasiado apresurado y urge terminarlo, es demasiado tosco cuando tenemos sexo" y "el sexo con se ha vuelto una tarea para mí".

\section{Procedimiento y análisis de datos:}

para aplicar los cuestionarios a la muestra se acudió a lugares como oficinas, escuelas, parques - entre otros- donde se podían encontrar a personas que de manera voluntaria y habiendo reconocido experimentar pasión romántica contestaran anónima y confidencialmente a la batería de medidas señaladas previamente. Ante dudas durante la aplicación se dio respuesta inmediata, asimismo, se puso a disposición de los participantes sus resultados personales. Para poder responder a la pregunta principal de la presente investigación se procedió a realizar una serie de análisis de correlación producto-momento de Pearson entre las fases de la pasión - atracción, enamoramiento, amor obsesivo y amor desesperado- con Phsc, personalidad sexual, comunicación emocional de la pasión, estrategias de seducción y satisfacción sexual por separado para cada sexo.

\section{Resultados}

Realizados los análisis pertinentes, a continuación se presentan los resultados por fase para hombres y mujeres respectivamente.

Cuando los hombres sienten atracción, tienden a creer (Phsc's) en la importancia de la emoción y de la felicidad que conlleva — reflexión emocional—, en que la pasión debe favorecer la intensidad en besos, abrazos y caricias - contacto físico intenso-, la expresión a través de la entrega sin medida - expresión abierta y plena-, creer que se debe soñar, desear e imaginar cómo seducir a su OP para que sus deseos se vuelvan hechos - deseo e imaginación-. Pero a la vez evaluar sus consecuencias de manera que haya responsabilidad y regulación - control-, sin olvidar el disfrute que implica. Al respecto, las mujeres muestran gran similitud en cuanto a sus creencias, excepto que ellas no creen que la PR deba controlarse. En cuanto a la personalidad sexual percibida en su OP, se observa que cuando las mujeres sienten atracción ven a dicha persona más adorable y amorosa -involucrado emocionalmente-, apasionada y atrayente - atractivo sexual-, conquistador y lujurioso - dispuesto eróticamente- y más masculino - orientación de género-. Mientras que los hombres también perciben a sus OP como involucradas emocionalmente y atractivas sexualmente, aunado a poseer una disposición erótica. En cuanto a las estrategias de seducción, tanto hombres como mujeres cuando sienten atracción ejecutan las cuatro estrategias en las que hay caricias en los genitales y excitación - expresión sexual-, mantener su interés y coquetearle — búsqueda de cercanía-, a través de la libertad y seguridad personales — desenvolvimiento y expresión libre - y siendo cariñoso y detallistas enamoramiento-. Y por último, ambos sexos experimentan satisfacción sexual (ver tabla 1).

En cuanto al enamoramiento, tanto los hombres como las mujeres que sienten antojo, excitación y fantasías por su OP creen que ante la PR se debe desear e imaginar a la persona, expresarse abierta y plenamente para lograr realizar sus deseos, tener contacto físico intenso y disfrutar sin restricciones. Hay que señalar que al respecto de esta variable, únicamente los hombres enamorados creen que deben reflexionar sobre la naturalidad e importancia de lo que sienten. Quienes están enamorados — hombres y mujeresperciben a su OP como dispuesto eróticamente, atractivo sexual e involucrado emocionalmente. Las mujeres, por su parte, perciben a su OP como promiscuo -infiel y mujeriego-. Asimismo, en seducción, ambos sexos mostraron ejecutar las cuatro las estrategias evaluadas y en la satisfacción sexual ambos perciben disfrute y excitación (ver tabla 2).

Respecto a las fases negativas, el comportamiento de las variables dista en forma interesante de las positivas. En términos de las Phsc se observa que cuando ellos experimentan un amor obsesivo, creen que la PR implica el estar con el OP lo más posible 
Tabla 1.

Análisis de correlación para la fase de atracción.

\begin{tabular}{|c|c|c|c|}
\hline & Factores & Hombres & Mujeres \\
\hline \multirow{6}{*}{ Phsc } & Expresión abierta y plena & $.435^{* *}$ & $.275^{\star *}$ \\
\hline & Deseo e imaginación & $.391^{* *}$ & $.218^{* *}$ \\
\hline & Contacto físico intenso & $.496^{* *}$ & $.298^{\star *}$ \\
\hline & Reflexión emocional & $.523^{\star *}$ & $.306^{* *}$ \\
\hline & Disfrute & $.323^{* *}$ & $.239^{* *}$ \\
\hline & Control & $.356^{* *}$ & - \\
\hline \multirow{7}{*}{ Personalidad sexual } & Promiscuo & - & - \\
\hline & Involucrado emocionalmente & $.573^{* *}$ & $.554^{* *}$ \\
\hline & Atractivo sexual & $.573^{* *}$ & $.398^{\star *}$ \\
\hline & Dispuesto eróticamente & $.316^{* *}$ & $.214^{* *}$ \\
\hline & Orientación de género & - & $.214^{* *}$ \\
\hline & Restricción sexual & - & - \\
\hline & Comunicación emocional de la pasión & - & - \\
\hline \multirow{4}{*}{ Estrategias de seducción } & Expresión sexual & $.374^{* *}$ & $.316^{* *}$ \\
\hline & Búsqueda de cercanía y permanencia con la pareja & $.472^{* *}$ & $.378^{* *}$ \\
\hline & Desenvolvimiento y expresión libre & $.349^{* *}$ & $.362^{\star *}$ \\
\hline & Enamoramiento & $.577^{\star *}$ & $.469^{* *}$ \\
\hline \multirow{2}{*}{ Satisfacción sexual } & Satisfacción & $.316^{* *}$ & $.237^{\star *}$ \\
\hline & Insatisfacción & - & - \\
\hline
\end{tabular}

Fuente: elaboración propia

Tabla 2.

Análisis de correlación para la fase de enamoramiento.

\begin{tabular}{|c|c|c|c|}
\hline & Factores & Hombres & Mujeres \\
\hline \multirow{6}{*}{ Phsc } & Expresión abierta y plena & $.411^{\star *}$ & $.359^{* *}$ \\
\hline & Deseo e imaginación & $.476^{\star *}$ & $.370^{* *}$ \\
\hline & Contacto físico intenso & $.377^{\star *}$ & $.279^{* *}$ \\
\hline & Reflexión emocional & $.287^{\star *}$ & - \\
\hline & Disfrute & $.303^{\star *}$ & $.286^{* *}$ \\
\hline & Control & - & - \\
\hline \multirow{7}{*}{ Personalidad sexual } & Promiscuo & - & $.246^{* *}$ \\
\hline & Involucrado emocionalmente & $.421^{* *}$ & $.257^{\star *}$ \\
\hline & Atractivo sexual & $.443^{\star *}$ & $.443^{\star *}$ \\
\hline & Dispuesto eróticamente & $.444^{* *}$ & $.485^{* *}$ \\
\hline & Orientación de género & - & - \\
\hline & Restricción sexual & - & - \\
\hline & Comunicación emocional de la pasión & - & - \\
\hline
\end{tabular}


(continuación)

\begin{tabular}{|c|c|c|c|}
\hline \multirow{4}{*}{ Estrategias de seducción } & Expresión sexual & $.494^{\star *}$ & $.426^{* *}$ \\
\hline & Búsqueda de cercanía y permanencia con la pareja & $.477^{\star *}$ & $.368^{* *}$ \\
\hline & Desenvolvimiento y expresión libre & $.305^{\star *}$ & $.385^{* *}$ \\
\hline & Enamoramiento & $.390^{* *}$ & $.309^{* *}$ \\
\hline \multirow{2}{*}{ Satisfacción sexual } & Satisfacción & $.348^{* *}$ & $.308^{* *}$ \\
\hline & Insatisfacción & - & - \\
\hline
\end{tabular}

Fuente: elaboración propia

dando besos y abrazos, estar dispuesto a entregarse y realizar su deseo, así como fantasear con la persona amada e imaginarse haciendo de todo con ella. Los hombres obsesivos perciben a su OP como más promiscua a la vez que resitringida sexualmente y femenina/hembra - orientación de género-, mientras que las mujeres de esta fase perciben a su OP casto e inmaculado - restringido sexualmente-. En términos de la comunicación emocional de la PR son únicamente los hombres quienes sienten pasión a partir de lo que interpretan de las conductas verbales y no verbales de sus OP. En la medida en que hombres se ubican como más obsesivos al expresar su pasión, estos sienten mayor insatisfacción y algo de satisfacción sexual, mientras que las mujeres solo sienten insatisfacción (ver tabla 3).

Cuando el amante apasionado se ubica en la fase caracterizada por la emisión de conductas desesperadas para lograr la reciprocidad en su OP, se evidencian comportamientos como investigar la forma de seducirle a cualquier costo, perseguirla para obtener contacto - entre otras características-, tiende

Tabla 3.

Análisis de correlación para la fase de amor obsesivo.

\begin{tabular}{|c|c|c|c|}
\hline & Factores & Hombres & Mujeres \\
\hline \multirow{6}{*}{ Phsc } & Expresión abierta y plena & $.265^{\star *}$ & - \\
\hline & Deseo e imaginación & $.225^{\star *}$ & - \\
\hline & Contacto físico intenso & $.326^{* *}$ & - \\
\hline & Reflexión emocional & - & - \\
\hline & Disfrute & - & - \\
\hline & Control & - & - \\
\hline \multirow{7}{*}{ Personalidad sexual } & Promiscuo & $.569^{* *}$ & - \\
\hline & Involucrado emocionalmente & - & - \\
\hline & Atractivo sexual & - & - \\
\hline & Dispuesto eróticamente & - & - \\
\hline & Orientación de género & $.296^{\star *}$ & - \\
\hline & Restricción sexual & $.565^{\star *}$ & $.265^{\star *}$ \\
\hline & Comunicación emocional de la pasión & $.233^{\star *}$ & - \\
\hline \multirow{4}{*}{ Estrategias de seducción } & Expresión sexual & - & - \\
\hline & Búsqueda de cercanía y permanencia con la pareja & - & - \\
\hline & Desenvolvimiento y expresión libre & - & - \\
\hline & Enamoramiento & - & - \\
\hline \multirow{2}{*}{ Satisfacción Sexual } & Satisfacción & $.427^{\star *}$ & - \\
\hline & Insatisfacción & $.584^{* *}$ & .219 \\
\hline
\end{tabular}

Fuente: elaboración propia 
- cuando es hombre- a creer en que hay que tener contacto físico intenso - estar junto a la persona lo más posible-, abrazar y besar a la persona y realizar su deseo - expresión abierta y plena-, así como fantasear con la persona amada, soñar con tener contacto íntimo con la persona - deseo e imaginación-. A medida que los hombres y mujeres sienten más amor desesperado, perciben que su OP es más promiscuo y restringidos sexualmente - más alto en hombres-. Para los hombres también resultó significativa la relación entre el amor desesperado y el rasgo de orientación de género - es decir, percibir a la mujer como "hembra"-. Al igual que en la fase anterior, son los hombres en amor desesperado los que sienten pasión debido a las conductas no verbales y verbales de sus OP. Por último, se observa que a más amor desesperado, más insatisfacción - en hombres y mujeres-y algo de satisfacción en hombres (ver tabla 4).

\section{Discusión}

La investigación realizada buscó - a partir de las relaciones entre las fases de la PR con las Phsc de la PR, la personalidad sexual, la comunicación emocional, las estrategias de seducción y la satisfacción sexual en hombres y mujeres- caracterizar a la atracción, enamoramiento, amor obsesivo y amor desesperado, para con ello lograr un conocimiento más profundo de ellas como fases de la PR.

Con base en esta lógica y descritos los resultados por fase y por sexo de una forma específica y clara. La discusión en curso estará inicialmente guiada hacia la definición particular de cada una de las fases en forma integral, a partir de las coincidencias que los datos reportan en su experiencia; para luego examinar las diferencias por sexo.

Tabla 4.

Análisis de correlación para la fase de amor desesperado.

\begin{tabular}{|c|c|c|c|}
\hline & Factores & Hombres & Mujeres \\
\hline \multirow{6}{*}{ Phsc } & Expresión abierta y plena & $.254^{\star *}$ & - \\
\hline & Deseo e imaginación & $.241^{\star \star}$ & - \\
\hline & Contacto físico intenso & $.312^{\star *}$ & - \\
\hline & Reflexión emocional & - & - \\
\hline & Disfrute & - & - \\
\hline & Control & - & - \\
\hline \multirow{7}{*}{ Personalidad sexual } & Promiscuo & $.629^{\star *}$ & $.311^{* *}$ \\
\hline & Involucrado emocionalmente & - & - \\
\hline & Atractivo sexual & - & - \\
\hline & Dispuesto eróticamente & - & - \\
\hline & Orientación de género & $.388^{\star *}$ & - \\
\hline & Restricción sexual & $.569^{* *}$ & $.306^{* *}$ \\
\hline & Comunicación emocional de la pasión & $.230^{\star *}$ & - \\
\hline \multirow{4}{*}{ Estrategias de seducción } & Expresión sexual & - & - \\
\hline & Búsqueda de cercanía y permanencia con la pareja & - & - \\
\hline & Desenvolvimiento y expresión libre & - & - \\
\hline & Enamoramiento & - & - \\
\hline \multirow{2}{*}{ Satisfacción sexual } & Satisfacción & $.372^{* *}$ & - \\
\hline & Insatisfacción & $.612^{\star *}$ & $.405^{\star *}$ \\
\hline
\end{tabular}

${ }^{* *} p<0,01$

Fuente: elaboración propia 
Cuando una persona se siente en la fase de la atracción cuya esencia radica en la felicidad, goce, emoción, ilusión, plenitud sentida ante el pensamiento o presencia del OP; cree y valora a la experiencia de la pasión como algo positivo que debe reflexionarse sobre su importancia, que involucra contacto físico como besos, caricias y abrazos, así como la expresión plena que involucra gran deseo e imaginación. Esto se ve apoyado por un sinnúmero de autores y legos que reconocen que durante la atracción se manifiesta un impulso dominante de formar y mantener una relación personal duradera y positiva (Baumeister y Leary, 1995). Aunado a ello, se experimenta gran placer ante la experiencia de conocer e interactuar con otra persona con el propósito - inherente- de pertenencia profunda y expresión emocional en dicha relación que va tornándose significativa. Cuando los participantes le confieren valor a esta experiencia a través de sus creencias es porque - de acuerdo a Hill, 1987- piensan que la atracción les provee de un equilibrio óptimo en su vida así como la oportunidad de recibir atención y apoyo emocional. Cuando el individuo se encuentra en esta fase, tiende a percibir a su OP como alguien con una personalidad sexual atrayente y apasionada, es decir, alguien sexualmente atractivo y con gran potencial de involucrarse emocionalmente - adorable y amoroso-. Esto tiene sentido si se piensa que dichos atributos son facilitadores de la conexión inicial entre ambas personas de manera que pueda desarrollarse la atracción intensa que involucra la idealización del otro en un contexto erótico (Jankowiak, 1995; Fisher, Aron, Mashek, Haifang, y Brown, 2002), el deseo por la intimidad y la expectativa placentera de mantener la experiencia por cierto tiempo. En este contexto, quienes están pasando por la atracción hacen uso de una amplia gama de estrategias de seducción que van desde ser amable y detallista con el OP, pasando por llamar su atención, coquetear, sentirse seguro durante el ejercicio de la seducción, hasta buscar excitarle, y provocarse sensaciones placenteras. Esto repercute positivamente en el gozo percibido respecto a la vida sexual ya que se considera excitante, disfrutable y se evalúa al compañero sexual como alguien "maravilloso".

Cuando se encuentra el individuo en el enamoramiento, sienten el deseo intenso de explorar, tocar, relacionarse sexualmente, y la seducción como el estremecimiento surgen ante la idea o presencia del OP. Esta experiencia se complementa con una serie de Phsc a las que la persona tiene apego e indican que ante la pasión se debe fantasear con la persona en forma ilimitada, entregarse y expresar abierta y plenamente lo que se siente para lograr realizar sus deseos, tener contacto físico intenso y disfrutar sin restricciones. Esto refleja una interacción fluida entre lo que se ha introyectado de la cultura y lo que las personas sienten al enamorarse, como el deseo sexual intenso por consumarlo en la entrega sin medida e incluye: enamoramiento, la necesidad de estar cerca del otro constantemente, irracionalidad, amor, ímpetu, la intrusión obsesiva, la búsqueda desesperada por tener cercanía física continua, la alegría, el erotismo y la sensualidad (Díaz Loving y Sánchez Aragón, 2002), o un estado de intenso anhelo de unión con el otro (Hatfield y Rapson, 1996). Quienes están enamorados perciben a su OP como dispuesto eróticamente, atractivo sexual e involucrado emocionalmente como en el caso de la atracción, pero con un énfasis distinto pues en esta fase el erotismo predomina y se vive como una emoción caliente (Hatfield y Rapson, 1996) que pretende satisfacer las necesidades de felicidad apasionada, euforia, excitación, risa y satisfacción (Fehr, 1993). En lo tocante a las estrategias de seducción, la persona enamorada que siente prisa y precipitación por el placer y un anhelo por la pasión erótica (Fehr, 1993), ejecuta las cuatro las estrategias evaluadas - al igual que en la atracción- pero con coeficientes de correlación un poco más altos en la expresión sexual y la búsqueda de cercanía y permanencia con la pareja, lo que da coherencia a lo hallado en esta fase. Durante el enamoramiento, el componente sexual se hace más presente (Sánchez Aragón et al., 2006; Sánchez Aragón, 2007ab), el despliegue de estas estrategias se vuelve decisivo y su variedad contribuye al goce sexual de la persona y de su OP.

El amante obsesivo es definido como aquella persona que se percibe con angustia, tristeza y desesperación ante el alejamiento o separación del ser amado, sus expectativas de felicidad, tranquilidad y valía están en función de su OP; por lo tanto, experimenta invalidez ante la ausencia del objeto de amor. A diferencia de aquellos ubicados en las fases positivas de la pasión que apoyaban la mayor parte de las Phsc y con coeficientes de correlación más fuertes, aquí no hubo consenso entre hombres y mujeres respecto a sus creencias. Es necesario recordar que quienes puntúan más en el amor obsesivo son personas que están experimentando cierta falta de mutualidad, lo cual los hace ávidos de atención, contacto y correspondencia (Bratslavsky, Baumeister, y Sommer, 1998; 
Hatfield y Rapson, 1996) esto les provoca sufrimiento y dolor. Ante este escenario, la forma en la que los amantes obsesivos perciben a sus OP es interesante, tienden a verlos con atributos como ser inmaculado y puro. Estos rasgos resultan congruentes en estas personas ya que al no encontrar reciprocidad y poseer un exceso de preocupación, sentimientos de vacio, desesperanza y una urgencia de tener a su OP, lo miran restringido sexualmente (Hatfield y Rapson, 1996). Por lo tanto, en la medida en que estas personas se ubican como apasionados obsesivos, no tienen la posibilidad de desplegar estrategias de seducción que conlleven a la insatisfacción sexual. Estos datos muestran como el amor no correspondido revela un lado oscuro de las relaciones románticas; hay un desbalance en el grado de necesidad o deseo por una relación, lo cual trae consecuencias destructivas para los miembros involucrados como sentimientos de frutración e incertidumbre, por un lado; y culpa y remordimiento, por otro (Bratslavsky et al., 1998).

Finalmente, quien siente amor desesperado busca en forma persistente la interacción con su OP, incluyendo estrategias de persecución, imponiéndole su presencia, bromeándole sobre temas sexuales con el fin de impedir que algo se interponga entre ellos y así lograr la atención o correspondencia. En cuanto a la percepción de su OP, estos amantes lo ven como promiscuo, y - al igual que los obsesivos- restringido sexualmente, es decir, limitado para el sexo y la aventura, lo cual se une a la insatisfacción sexual. Esto se apoya en la falta de correspondencia y la presencia de hostigamiento o hasta coerción, lo cual hace más que imposible la unión armónica con el OP. Esta situación le trae al amante desesperado insatisfacción sexual que puede conllevar consecuencias emocionales negativas (Muehlenhard, Goggins, Jones, y Satterfield, 1991).

\section{Diferencias sexuales en las fases de la pasión}

En cuanto a las diferencias entre los sexos, se encuentra que, a más atracción, los hombres creen en el control durante la experiencia. Al respecto, estudios relativos a la regulación emocional han demostrado como los hombres tienden a usar más estrategias como la supresión expresiva y la reapreciación cognoscitiva para emociones como la felicidad (Sánchez Aragón y Méndez Canales, 2011). Por su parte, las mujeres ven a su OP como varonil y masculino, lo cual resulta natural; apoyado en una lógica evolutiva en donde las mujeres tienden a buscar a hombres con cuerpos atlénticos y fuertes con capacidades de dominancia sexual (Buss, 2016).

En el enamoramiento los hombres, a diferencia de las mujeres, tienden a creer en la reflexión respecto a la importancia e impacto de la pasión en sus vidas lo cual puede resultar congruente con el peso que la cultura ejerce en los hombres, autorizándoles un ejercicio más libre de ésta (Díaz Loving y Rivera Aragón, 2010). En esta fase ellas perciben a su OP como promiscuo -infiel y mujeriego-, atributo asignado tradicionalmente a los hombres y que pueden resultar "útiles" durante el cortejo y el romance (Díaz Guerrero, 2004).

En la fase del amor obsesivo, también se observan diferencias interesantes. En cuanto a las Phsc los hombres tienden a creer más que se debe tener contacto físico intenso, una expresión abierta y plena, seguida del deseo y la imaginación cuando se siente PR. Aquí son únicamente ellos quienes cognoscitivamente muestran una preocupación por el valor de las manifestaciones positivas de dicha experiencia, quizá enfatizada por la falta de mutualidad y sobrevaloración de algo que no pueden poseer (Sánchez Aragón, 2007b) dada la naturaleza de esta fase. Cuando los hombres se perciben más obsesivos, ven a su OP como promiscua y con orientación de género, es decir, adúltera a la vez que "hembra", lo cual podría ser incluso- debido a la gran elaboración cognoscitiva de estos amantes, quienes al fantasear, imaginar y concentrar sus pensamientos voluntaria o involuntariamente en su OP, pueden depositar en ella no solo su felicidad como lo indica Sánchez Aragón (2007b), sino también sus más íntimos deseos; en donde el OP juega los papeles y tiene los atributos que al participante le resultan más apropiados a lo que desea. Por último, en cuando a la satisfacción sexual son los hombres quienes muestran cierta satisfacción sexual, quizá más mental que real.

En el amor desesperado, el apego a las creencias es similar al caso del amor obsesivo, lo cual es entendible, dada la carencia de mutualidad estos hombres refuerzan la importancia de lo que no tienen y creen en ello casi como aquellos en las fases positivas. Ellos ven a su OP como "hembra" lo cual se apoya en lo reportado por Buss (2016) quien señala que los hombres buscan alguien con "alta capacidad de reproducción" y cuyas características físicas sean atractivas y 
les garanticen sexo gratificante. Esto se complementa con el hecho de que estos apasionados románticos, tienden a interpretar — aún las señales no dirigidas a ellos- como estimulantes para reforzar su pasión, lo cual les puede crear una confusión a la vez que sufrimiento pues no hay correspondencia entre su sentir y aquello que elaboran en su pensamiento (Bratslavsky et al., 1998), esto repercute en una satisfacción sexual -quizá- ficticia.

La presente investigación ofrece un panorama más amplio de lo que es y cómo se viven las fases del amor pasional (Sánchez Aragón, 2007b) en una muestra de población mexicana. No obstante, se reconoce la necesidad de examinar más profundamente el comportamiento de dichas fases por grupos de edad, integrando otras variables vinculadas y teniendo en consideración más características de las muestras, como la conducta sexual. Por medio de los datos se logró tener, por un lado, la caracterización de la atracción, el enamoramiento, el amor obsesivo y el amor desesperado; y por otro, una interesante exploración de lo que distingue a hombres y mujeres respecto a la experiencia de dichas fases desde el punto de vista cultural. Los datos aquí presentados son una contribución en el entendimiento de la PR como un universal cultural en el contexto de la cultura mexicana.

\section{Referencias}

Álvarez-Gayou, J. (1987). Elementos de sexología. México: Interamericana.

Baumeister, R. F. and Leary, M. R. (1995). The need to belong: Desire for interpersonal attachments as a fundamental human motivation. Psychology Bulletin, 117, 498-529. doi: 10.10372F0033-2909.117.3.497

Bratslavsky, E., Baumeister, R. F., y Sommer, K. L. (1998). To love or Be loved in Vain: The Trials and Tribulations of Unrequited Love. In B. H. Spitzberg and W. R. Cupach. (Eds.). The Dark Side of Close Relationships. NJ: Lawrence Erlbaum Associates Inc.

Brehm, B. (1985). Intimate Relationships. USA: McGrawHill.

Byers, E.S. (2005). Relationship satisfaction and sexual satisfaction: A longitudinal study of individuals in longterm relationships. The Journal of Sex Research, 42, 113-118. doi: 10.1080/00224490509552264.

Buss, D. (2016). The evolution of desire. New York: Basic Books.
Caldíz, L. y Resnicoff, D. (1997). Sexo, mujer y fin de siglo: la intimidad redescubierta. Argentina: Paidós.

Carrasco Chávez, E. (2010). Seducción: Una forma de expresión emocional femenina. Tesis. Universidad Nacional Autónoma de México.

Collins, J. C. and Gregor, T. (1995). Boundaries of Love. In W. Jankowiak. (Ed.). Romantic Passion: A Universal experience? New York: Columbia University Press.

Cruz Vargas, D. J. (2018). Personalidad sexual, Comunicación emocional, Estrategias de regulación de la pasión y Cercanía como determinantes de la Satisfacción sexual en la pareja. Tesis. Universidad Nacional Autónoma de México.

Díaz Guerrero, R. (1994). La psicología del mexicano: descubrimiento de la etnopsicología. México: Trillas.

Díaz Guerrero, R. (2004). Psicología del Mexicano: descubrimiento de la etnopsicología. México: Trillas.

Díaz Loving, R. y Rivera Aragón, S. (2010). Relaciones sustentables: el papel del amor y otras especias en la promoción de parejas positivas. En Díaz Loving, R. y Rivera Aragón, S. (Comp.). Antología Psicosocial de la Pareja: Clásicos y Contemporáneos. México: Miguel Ángel Porrúa y AMEPSO.

Díaz Loving, R. y Sánchez Aragón, R. (2002). Psicología del Amor: Una visión integral de la relación de pareja. México: Miguel Ángel Porrúa.

Fehr, B. (1993). How do I love thee? Let me consult my prototype. In S. Duck. (Ed.). Individuals in Relationships: Understanding Relationship Processes Series, 1 (87120). Newbury Park, CA: Sage.

Fisher, H. E., Aron, A., Mashek, D., Haifang, L., and Brown, L. L. (2002). Defining the Brain Systems of Lust, Romantic Attraction and Attachment. Archives of Sexual Behavior, 31 (5), 413-419. doi: 10.1023/A:1019888024255.

Fletcher, G. J. O. and Kininmonth, L. (1991). Interaction in close relationships and social cognition. In G. J. O. Fletcher and F. D. Fincham. (Eds.). Cognition in close relationships. Hillsdale, NJ: Lawrence Erlbaum Associates Inc.

García Rodríguez, G. (2007). Conducta sexual: un modelo psicosocial. Tesis. Universidad Nacional Autónoma de México.

Guerrero, L. K., Andersen, P.A., and Afifi, W.A. (2017). Close Encounters. Communication in Relationships. Thousand Oaks: Sage Publications.

Hatfield, E. (1988). Passionate Love and Companionate Love. In R. Sternberg and M. Barnes. (Eds.). The Psychology of Love. New Haven: Yale University Press. 
Hatfield, E. and Rapson, R. (1996). Love and sex: cross-cultural perspectives. USA: Allyn and Bacon.

Hatfield, E., Rapson, R., and Martel, L. D. (2010). Passionate Love and Sexual Desire. En S. Kitayama and d. Cohen. (Eds.). Handbook of Cultural Psychology. New York: The Guilford Press.

Hill, C. A. (1987). Affiliation motivation: people who need people...but in different ways. Journal of Personality and Social Psychology, 52, 1008-1018. doi: 10.1037//0022-3514.52.5.1008.

Hinton, A. (1993). Prologomenon to a Processual Approach to the Emotions. Ethnos, 21(4), 1-22. doi: 10.1525/ eth.1993.21.4.02a00030.

Hudson, W. W. (1997). The WALMYR Assessment Scales Scoring Manual. Tallahassee: WALMYR Publishing Co.

Jankowiak, W. (1995). Introduction. In W. Jankowiak. (Ed.). Romantic Passion: A Universal experience? New York: Columbia University Press.

Jankowiak, W. and Fischer, E. (1992). A Cross-Cultural Perspective on Romantic Love. Ethnology, 31(2), 149-155. doi: $10.2307 / 3773618$.

Lee, J. A. (1976). Lovestyles. London: Dent \& Sons.

Lucker, G. W. (2002). La cultura y la psicología social. En C. Kimble, E. Hirt, R. Díaz- Loving, H. Hosch, G. Luker y M. Zárate. (Comp.). Psicología Social de la Américas (pp. 507-5531). México: Pearson Educación.

Metts, S. y Cupach, W. R. (1991). Sexuality and Communication in Close Relationships. In K. McKinney and. S. Sprecher (Eds.). Sexuality in Close Relationships. Hillsdale: Lawrence Erlbaum Associates, Publishers.

Muehlenhard, C. L., Goggins, M F., Jones, J. M., y Satterfield, A. T. (1991). Sexual Violence and Coercion in Close Relationships. In K. McKinney and S. Sprecher. (Eds.) Sexuality in Close Relationships. Hillsdale: Lawrence Erlbaum Associates, Publishers.

Paredes, J. y Pinto, B. (2009). Imagen corporal y satisfacción sexual. Ajayu, 7(1), 28-47.

Person, E. S. (2007). Dreams of Love and Fateful Encounters: The Power of Romantic Passion. Washington: American Psychiatric Publishing, Inc.

Sánchez Aragón, R. (2000). Valoración empírica de la teoría bio-psico-sociocultural de la relación de pareja. Tesis. Universidad Nacional Autónoma de México.

Sánchez Aragón, R. (2006). Validación de una escala de evaluación subjetiva de la experiencia pasional y su relación con el amor pasional. En Sánchez Aragón, R., Díaz Loving, R. y Rivera Aragón, S. (Eds.). La Psicología Social en México. México: AMEPSO y UJAT.
Sánchez Aragón, R. (2007a). Significado Psicológico del Amor Pasional: lo claro y lo oscuro. Revista Interamericana de Psicología, 41(3), 391-402.

Sánchez Aragón, R. (2007b). Pasión Romántica: más allá de la intuición, una ciencia del amor. Miguel Ángel Porrúa.

Sánchez Aragón, R. (2012). Comunicación Emocional en las Relaciones Románticas: Validación de una Medida en México. Interpersona, 6(2), 211-226. doi: 10.5964/ijpr. v6i2.102.

Sánchez Aragón, R. Cruz Martínez, L. Ma. y Retana Franco, B. E. (2006). ¿Cómo Soy en el Ámbito Sexual y cómo Percibo a mi Objeto de Pasión? En Sánchez Aragón, R., Díaz Loving, R. y Rivera Aragón, S. (Eds.). La Psicología Social en México. Mexico: AMEPSO y UJAT.

Sánchez Aragón, R., Jiménez Rodríguez, B. y Martínez Cruz, R. (2013). Creencias sobre la expresión de la pasión romántica en la cultura mexicana. Revista Latinoamericana de Psicología Social Ignacio Martín-Baró, 2(1), 111-136. Recuperado de: www.rimb.cl/sanchez_et_ al.html.

Sánchez Aragón, R. y Mendez Canales, R. (2011). Elementos Mediadores de la Felicidad y Bienestar Subjetivo de Hombres y Mujeres. Revista Costarricense de Psicología, 30(45-46), 51-76.

Sanz, J. R. (1995). Psicoerotismo femenino y masculino, para unas relaciones placenteras, autónomas y justas. Barcelona: Kairós.

Schmitt, D.P., and Buss, D.M. (2000). Sexual Dimensions of Person Description: Beyond or Subsumed by the Big Five? Journal of Research in Personality, 34, 141-177. doi: 10.1006/jrpe.1999.2267.

Sternberg, R. J. (1986). Triangular theory of love. Psychological Review, 93(2), 119-135. doi: 10.1037//0033295x.93.2.119.

Troy, A. B. (2005). Romantic Passion as Output from a Self-regulating, Intimacy-Seeking System: A Model for Understanding Passionate Love. Psychological Reports, 96, 655-675. doi: 10.2466/pr0.96.3.655-675.

Valdés, R. M., Sapién, L. J. y Córdoba, I. D.(2004). Significados de satisfacción sexual en hombres y mujeres de la zona metropolitana. Psicología y Ciencia Social, 6(1), 34-48.

Villanueva Orozco, G. B. T. (2004). De la atracción al acoso: ¿tipos o fases del amor pasional? Tesis. Universidad Nacional Autónoma de México.

Walster, E. and Walster, G. W. (1978). A New look at love. Reading: Addison-Wesley. 
Yela, C. (2000). Predictors and Factors related to loving and sexual satisfaction for men and women. European Review of Applied Psychology, 49, 235-242.

Young, M., Denny, G., Young, T., and Luquis, R. (2000). Sexual satisfaction among married women. American Journal of Health Studies, 16(2), 73-84.doi: 10.2466/ pro.86.3.1107-1122.
Ziherl, S. and Masten, R. (2010). Differences in predictors of sexual satisfaction and in sexual satisfaction between female and male university students in Slovenia. Psychiatria Danubina, 22(3), 425-429. 